Pacific

Journal of

Mathematics

\title{
RADIAL SOLUTIONS OF NON-ARCHIMEDEAN PSEUDODIFFERENTIAL EQUATIONS
}

AnATOLy N. KochubeI

Volume 269 No. 2

June 2014 


\title{
RADIAL SOLUTIONS OF NON-ARCHIMEDEAN PSEUDODIFFERENTIAL EQUATIONS
}

\author{
ANATOLY N. Kochubei
}

\begin{abstract}
We consider a class of equations with the fractional differentiation operator $D^{\alpha}, \alpha>0$, for complex-valued functions $x \mapsto f\left(|x|_{K}\right)$ on a non-Archimedean local field $K$ depending only on the absolute value $|\cdot|_{K}$. We introduce a right inverse $I^{\alpha}$ to $D^{\alpha}$, such that the change of an unknown function $u=I^{\alpha} v$ reduces the Cauchy problem for an equation with $D^{\alpha}$ (for radial functions) to an integral equation whose properties resemble those of classical Volterra equations. This contrasts much more complicated behavior of $D^{\alpha}$ on other classes of functions.
\end{abstract}

\section{Introduction}

Pseudodifferential equations for complex-valued functions defined on a non-Archimedean local field are among the central objects of contemporary harmonic analysis and mathematical physics; see the monographs [Vladimirov et al. 1994; Kochubei 2001; Albeverio et al. 2010], and the survey [Zúñiga-Galindo 2011].

The simplest example is the fractional differentiation operator $D^{\alpha}, \alpha>0$, on the field $\mathbb{Q}_{p}$ of $p$-adic numbers (here $p$ is a prime number). It can be defined as a pseudodifferential operator with the symbol $|\xi|_{p}^{\alpha}$ where $|\cdot|_{p}$ is the $p$-adic absolute value or, equivalently, as an appropriate convolution operator.

Already in this case, as it was first shown by Vladimirov (see [Vladimirov et al. 1994]), properties of the $p$-adic pseudodifferential operator are much more complicated than those of its classical counterpart. It suffices to say that, as an operator on $L_{2}\left(\mathbb{Q}_{p}\right)$, it has a point spectrum of infinite multiplicity. Considering a simple "formal" evolution equation with the operator $D^{\alpha}$ in the $p$-adic time variable $t$, Vladimirov [2003] noticed that such an equation does not possess a fundamental solution.

At the same time, it was found in [Kochubei 2008] that some of the evolution equations of the above kind behave reasonably, if one considers only solutions

This work was supported in part by Grant No. 01-01-12 of the National Academy of Sciences of Ukraine under the program of joint Ukrainian-Russian projects.

MSC2010: 11S80, 35S10.

Keywords: fractional differentiation operator, non-Archimedean local field, radial functions, Cauchy problem. 
depending on $|t|_{p}$. This observation has led to the concept of a non-Archimedean wave equation possessing various properties resembling those of classical hyperbolic equations, up to the Huygens principle.

In this paper we consider the Cauchy problem for a class of equations like

$$
D^{\alpha} u+a\left(|x|_{p}\right) u=f\left(|x|_{p}\right), \quad x \in \mathbb{Q}_{p},
$$

assuming that a solution is looked for in the class of radial functions, $u=u\left(|x|_{p}\right)$; the precise definition of $D^{\alpha}$ and assumptions on $a, f$ are given below. This Cauchy problem is reduced to an integral equation resembling classical Volterra equations. It turns out that (1-1) and its generalizations considered on radial functions constitute $p$-adic counterparts of ordinary differential equations.

\section{Preliminaries}

2.1. Local fields. Let $K$ be a non-Archimedean local field, that is, a nondiscrete totally disconnected locally compact topological field. It is well known that $K$ is isomorphic either to a finite extension of the field $\mathbb{Q}_{p}$ of $p$-adic numbers (if $K$ has characteristic 0 ), or to the field of formal Laurent series with coefficients from a finite field, if $K$ has a positive characteristic. For a summary of main notions and results regarding local fields see, for example, [Kochubei 2001].

Any local field $K$ is endowed with an absolute value $|\cdot|_{K}$, such that $|x|_{K}=0$ if and only if $x=0,|x y|_{K}=|x|_{K} \cdot|y|_{K},|x+y|_{K} \leq \max \left(|x|_{K},|y|_{K}\right)$. Set

$$
O=\left\{x \in K:|x|_{K} \leq 1\right\}, \quad P=\left\{x \in K:|x|_{K}<1\right\} .
$$

Then $O$ is a subring of $K$, and $P$ is an ideal in $O$ containing such an element $\beta$ that $P=\beta O$. The quotient ring $O / P$ is actually a finite field; denote by $q$ its cardinality. We will always assume that the absolute value is normalized, that is $|\beta|_{K}=q^{-1}$. The normalized absolute value takes the values $q^{N}, N \in \mathbb{Z}$. Note that for $K=\mathbb{Q}_{p}$ we have $\beta=p$ and $q=p$; the $p$-adic absolute value is normalized.

Denote by $S \subset O$ a complete system of representatives of the residue classes from $O / P$. Any nonzero element $x \in K$ admits the canonical representation in the form of the convergent series

$$
x=\beta^{-n}\left(x_{0}+x_{1} \beta+x_{2} \beta^{2}+\cdots\right)
$$

where $n \in \mathbb{Z},|x|_{K}=q^{n}, x_{j} \in S, x_{0} \notin P$. For $K=\mathbb{Q}_{p}$, one may take $S=$ $\{0,1, \ldots, p-1\}$.

The additive group of any local field is self-dual; that is, if $\chi$ is a fixed nonconstant complex-valued additive character of $K$, then any other additive character can be written as $\chi_{a}(x)=\chi(a x), x \in K$, for some $a \in K$. Below we assume that $\chi$ is a 
rank zero character, that is $\chi(x) \equiv 1$ for $x \in O$, while there exists such an element $x_{0} \in K$ that $\left|x_{0}\right|_{K}=q$ and $\chi\left(x_{0}\right) \neq 1$.

This duality is used in the definition of the Fourier transform over $K$. Denoting by $d x$ the Haar measure on the additive group of $K$ (normalized in such a way that the measure of $O$ equals 1 ) we write

$$
\tilde{f}(\xi)=\int_{K} \chi(x \xi) f(x) d x, \quad \xi \in K,
$$

where $f$ is a complex-valued function from $L_{1}(K)$. As usual, the Fourier transform F can be extended from $L_{1}(K) \cap L_{2}(K)$ to a unitary operator on $L_{2}(K)$. If $\mathscr{F} f=\tilde{f} \in L_{1}(K)$, we have the inversion formula

$$
f(x)=\int_{K} \chi(-x \xi) \tilde{f}(\xi) d \xi
$$

2.2. Integration formulas. As in real analysis, there are many well known formulas for integrals of complex-valued functions defined on subsets of a local field. There exist even tables of such integrals [Vladimirov 2003]. Note that formulas for integrals on $\mathbb{Q}_{p}$ and its subsets, as a rule, carry over to the general case, if one substitutes the normalized absolute value for $|\cdot|_{p}$ and $q$ for $p$.

Here we collect some formulas used in this work. Let $n \in \mathbb{Z}, \alpha>0$.

$$
\int_{|x|_{K} \leq q^{n}}|x|_{K}^{\alpha-1} d x=\frac{1-q^{-1}}{1-q^{-\alpha}} q^{\alpha n}
$$

$$
\int_{|x|_{K}=q^{n}}|x-a|_{K}^{\alpha-1} d x=\frac{q-2+q^{-\alpha}}{q\left(1-q^{-\alpha}\right)}|a|_{K}^{\alpha}, \quad|a|_{K}=q^{n} .
$$

$$
\int_{|x|_{K} \leq q^{n}} \log |x|_{K} d x=\left(n-\frac{1}{q-1}\right) q^{n} \log q .
$$

$$
\int_{|x|_{K}=q^{n}} \log |x-a|_{K} d x=\left[\left(1-\frac{1}{q}\right) \log |a|_{K}-\frac{\log q}{q-1}\right]|a|_{K}, \quad|a|_{K}=q^{n} .
$$

$$
\int_{|x|_{K} \leq q^{n}} d x=q^{n} ; \int_{|x|_{K}=q^{n}} d x=\left(1-\frac{1}{q}\right) q^{n} .
$$

$$
\int_{\substack{|x|_{K}=q^{n} \\ x_{0}=k_{0}}} d x=q^{n-1}, \quad 0 \neq k_{0} \in S
$$

(the restriction $x_{0}=k_{0}$ is in the sense of the canonical representation (2-1)). 


$$
\int_{\substack{|x|_{K}=q^{n} \\ x_{0} \neq k_{0}}} d x=\left(1-\frac{2}{q}\right) q^{n} .
$$

2.3. Test functions and distributions. A function $f: K \rightarrow \mathbb{C}$ is said to be locally constant, if there exists such an integer $l$ that for any $x \in K$

$$
f\left(x+x^{\prime}\right)=f(x), \quad \text { whenever }\left|x^{\prime}\right| \leq q^{-l} .
$$

The smallest number $l$ with this property is called the exponent of local constancy of the function $f$.

Let $\mathscr{D}(K)$ be the set of all locally constant functions with compact supports; it is a vector space over $\mathbb{C}$ with the topology of double inductive limit

$$
\mathscr{D}(K)=\underset{N \rightarrow \infty}{\lim _{l \rightarrow \infty}} \lim _{l \rightarrow \infty} \mathscr{D}_{N}^{l}
$$

where $\mathscr{D}_{N}^{l}$ is the finite dimensional space of functions supported in the ball $B_{N}=$ $\left\{x \in K:|x| \leq q^{N}\right\}$ and having the exponents of local constancy $\leq l$. The strong conjugate space $\mathscr{D}^{\prime}(K)$ is called the space of Bruhat-Schwartz distributions.

The Fourier transform preserves the space $\mathscr{D}(K)$. Therefore the Fourier transform of a distribution defined by duality acts continuously on $\mathscr{D}^{\prime}(K)$. As in the case of $\mathbb{R}^{n}$, there exists a well developed theory of distributions over local fields; it includes such topics as convolution, direct product, homogeneous distributions etc (see [Vladimirov et al. 1994; Kochubei 2001; Albeverio et al. 2010]). In connection with homogeneous distributions, it is useful to introduce the subspaces of $\mathscr{D}(K)$ :

$$
\begin{gathered}
\Psi(K)=\{\psi \in \mathscr{D}(K): \psi(0)=0\}, \\
\Phi(K)=\left\{\varphi \in \mathscr{D}(K): \int_{K} \varphi(x) d x=0\right\} .
\end{gathered}
$$

The Fourier transform $\mathscr{F}$ is a linear isomorphism from $\Psi(K)$ onto $\Phi(K)$, thus also from $\Phi^{\prime}(K)$ onto $\Psi^{\prime}(K)$. The spaces $\Phi(K)$ and $\Phi^{\prime}(K)$ are called the Lizorkin spaces (of the second kind) of test functions and distributions respectively; see [Albeverio et al. 2010]. Note that two distributions differing by a constant summand coincide as elements of $\Phi^{\prime}(K)$.

\section{Fractional differentiation and integration operators}

3.1. Riesz kernels and operators generated by them. On a test function $\varphi \in \mathscr{D}(K)$, the fractional differentiation operator $D^{\alpha}, \alpha>0$, is defined as

$$
\left(D^{\alpha} \varphi\right)(x)=\mathscr{F}^{-1}\left[|\xi|_{K}^{\alpha}(\mathscr{F}(\varphi))(\xi)\right](x) .
$$


However $D^{\alpha}$ does not act on the space $\mathscr{D}(K)$, since the function $\xi \mapsto|\xi|_{K}^{\alpha}$ is not locally constant. On the other hand, $D^{\alpha}: \Phi(K) \rightarrow \Phi(K)$ and $D^{\alpha}: \Phi^{\prime}(K) \rightarrow \Phi^{\prime}(K)$; see [Albeverio et al. 2010], and that was a motivation to introduce these spaces.

The operator $D^{\alpha}$ can also be represented as a hypersingular integral operator:

$$
\left(D^{\alpha} \varphi\right)(x)=\frac{1-q^{\alpha}}{1-q^{-\alpha-1}} \int_{K}|y|_{K}^{-\alpha-1}[\varphi(x-y)-\varphi(x)] d y
$$

[Vladimirov et al. 1994; Kochubei 2001]. In contrast to (3-1), the expression in the right of (3-2) makes sense for wider classes of functions. Below we study this in detail for the case of radial functions.

The expression in (3-2) is in fact the convolution $f_{-\alpha} * \varphi$, where the Riesz kernel $f_{s}$, for complex $s \notin 1+\frac{2 \pi i}{\log q} \mathbb{Z}$, is defined first for $\operatorname{Re} s>0$ as

$$
f_{s}(x)=\frac{|x|_{K}^{s-1}}{\Gamma_{K}(s)}, \quad \Gamma_{K}(s)=\frac{1-q^{s-1}}{1-q^{-s}},
$$

and then extended meromorphically to the remaining nonzero values of $s$ as a distribution from $\mathscr{D}^{\prime}(K)$ :

$$
\left\langle f_{s}, \varphi\right\rangle=\frac{1-q^{-1}}{1-q^{s-1}} \varphi(0)+\frac{1-q^{-s}}{1-q^{s-1}}\left[\int_{|x|_{K}>1} \varphi(x) \frac{d x}{|x|_{K}^{1-s}}+\int_{|x|_{K} \leq 1}(\varphi(x)-\varphi(0)) \frac{d x}{|x|_{K}^{1-s}}\right],
$$

For $s=0$, we set $f_{0}(x)=\delta(x)$. For $s \in 1+\frac{2 \pi i}{\log q} \mathbb{Z}$, we define

$$
f_{S}(x)=\frac{1-q}{\log q} \log |x|_{K} .
$$

It is well known that $f_{s} * f_{t}=f_{s+t}$ in the sense of distributions from $\mathscr{D}^{\prime}(K)$, so long as $s, t, s+t \notin 1+\frac{2 \pi i}{\log q} \mathbb{Z}$. If these kernels are considered as distributions from $\Phi^{\prime}(K)$, then $f_{s} * f_{t}=f_{s+t}$ for all $s, t \in \mathbb{C}$ [Albeverio et al. 2010]. In view of this identity, it is natural to define the operator $D^{-\alpha}, \alpha>0$, setting

$$
\begin{aligned}
&\left(D^{-\alpha} \varphi\right)(x)=\left(f_{\alpha} * \varphi\right)(x)=\frac{1-q^{-\alpha}}{1-q^{\alpha-1}} \int_{K}|x-y|_{K}^{\alpha-1} \varphi(y) d y, \\
& \varphi \in \mathscr{D}(K), \alpha \neq 1,
\end{aligned}
$$

and

$$
\left(D^{-1} \varphi\right)(x)=\frac{1-q}{q \log q} \int_{K} \log |x-y|_{K} \varphi(y) d y .
$$

Then $D^{\alpha} D^{-\alpha}=I$ on $\mathscr{D}(K)$, if $\alpha \neq 1$. This remains valid on $\Phi(K)$ also for $\alpha=1$.

The notions and results above are well known; see [Vladimirov et al. 1994; Albeverio et al. 2010]. We now come to new phenomena, considering the case of radial functions. 
3.2. Operators on radial functions. Let $u$ be a radial function, that is $u=u\left(|x|_{K}\right)$, $x \in K$. (In order to make the notation concise, we identify the function $x \mapsto u\left(|x|_{K}\right)$ on $K$ with the function $|x|_{K} \mapsto u\left(|x|_{K}\right)$ on $q^{\mathbb{Z}}$. This abuse of notation will not lead to confusion.)

Let us find an explicit expression for $D^{\alpha} u, \alpha>0$. Below we write $d_{\alpha}=$ $\left(1-q^{\alpha}\right) /\left(1-q^{-\alpha-1}\right)$. For $x \in K$, we denote by $x_{0}$ the element from $S \subset O$ appearing in the representation (2-1).

Lemma 1. If a function $u=u\left(|x|_{K}\right)$ is such that

$$
\sum_{k=-\infty}^{m} q^{k}\left|u\left(q^{k}\right)\right|<\infty, \quad \sum_{l=m}^{\infty} q^{-\alpha l}\left|u\left(q^{l}\right)\right|<\infty,
$$

for some $m \in \mathbb{Z}$, then for each $n \in \mathbb{Z}$ the expression in the right-hand side of (3-2) with $\varphi(x)=u\left(|x|_{K}\right)$ exists for $|x|_{K}=q^{n}$, depends only on $|x|_{K}$, and

$$
\begin{array}{r}
\left(D^{\alpha} u\right)\left(q^{n}\right)=d_{\alpha}\left(1-\frac{1}{q}\right) q^{-(\alpha+1) n} \sum_{k=-\infty}^{n-1} q^{k} u\left(q^{k}\right)+q^{-\alpha n-1} \frac{q^{\alpha}+q-2}{1-q^{-\alpha-1}} u\left(q^{n}\right) \\
+d_{\alpha}\left(1-\frac{1}{q}\right) \sum_{l=n+1}^{\infty} q^{-\alpha l} u\left(q^{l}\right) .
\end{array}
$$

Proof. We find, using the ultrametric properties of the absolute value, that

$$
\left(D^{\alpha} u\right)(x)=d_{\alpha} \int_{|y|_{K} \geq|x|_{K}}|y|_{K}^{-\alpha-1}\left[u\left(|x-y|_{K}\right)-u\left(|x|_{K}\right)\right] d y .
$$

If $|y|_{K}=|x|_{K}$ and $y_{0} \neq x_{0}$, the integrand vanishes. Therefore, by (2-6),

$$
\begin{aligned}
\left(D^{\alpha} u\right)(x) & =d_{\alpha} \sum_{k=-\infty}^{n-1} \int_{|y-x|_{K}=q^{k}}|x|_{K}^{-\alpha-1}\left[u\left(q^{k}\right)-u\left(q^{n}\right)\right] d y \\
& +d_{\alpha} \sum_{l=n+1}^{\infty} \int_{|y|_{K}=q^{l}} q^{-l(\alpha+1)}\left[u\left(q^{k}\right)-u\left(q^{n}\right)\right] d y \\
& =d_{\alpha}\left(1-\frac{1}{q}\right) q^{-(\alpha+1) n} \sum_{k=-\infty}^{n-1} q^{k}\left[u\left(q^{k}\right)-u\left(q^{n}\right)\right] \\
& +d_{\alpha}\left(1-\frac{1}{q}\right) \sum_{l=n+1}^{\infty} q^{-\alpha l}\left[u\left(q^{l}\right)-u\left(q^{n}\right)\right] .
\end{aligned}
$$

It is clear from this expression that $\left(D^{\alpha} u\right)(x),|x|_{K}=q^{n}$, depends only on $|x|_{K}$. After elementary transformations we get (3-6). 
Definition. We say that the action $D^{\alpha} u, \alpha>0$, on a radial function $u$ is defined in the strong sense if the function $u$ satisfies (3-5), so that $D^{\alpha} u\left(|x|_{K}\right),|x|_{K} \neq 0$, is given by (3-6), and there exists the limit

$$
D^{\alpha} u(0) \stackrel{\text { def }}{=} \lim _{x \rightarrow 0} D^{\alpha} u\left(|x|_{K}\right)
$$

It is evident from (3-2) that $D^{\alpha}$ annihilates constant functions (recall that in $\Phi^{\prime}(K)$ they are equivalent to zero). Therefore $D^{-\alpha}$ is not the only possible choice of the right inverse to $D^{\alpha}$. In particular, we will use

$$
\left(I^{\alpha} \varphi\right)(x)=\left(D^{-\alpha} \varphi\right)(x)-\left(D^{-\alpha} \varphi\right)(0) .
$$

This is defined initially for $\varphi \in \mathscr{D}(K)$. It is seen from (3-3), (3-4), and the ultrametric property of the absolute value that

$$
\left(I^{\alpha} \varphi\right)(x)=\frac{1-q^{-\alpha}}{1-q^{\alpha-1}} \int_{|y|_{K} \leq|x|_{K}}\left(|x-y|_{K}^{\alpha-1}-|y|_{K}^{\alpha-1}\right) \varphi(y) d y, \quad \alpha \neq 1,
$$

and

$$
\left(I^{1} \varphi\right)(x)=\frac{1-q}{q \log q} \int_{|y|_{K} \leq|x|_{K}}\left(\log |x-y|_{K}-\log |y|_{K}\right) \varphi(y) d y .
$$

In contrast to (3-3) and (3-4), in (3-8) and (3-9) the integrals are taken, for each fixed $x \in K$, over bounded sets.

Let us calculate $I^{\alpha} u$ for a radial function $u=u\left(|x|_{K}\right)$. Obviously, $\left(I^{\alpha} u\right)(0)=0$ whenever $I^{\alpha}$ is defined.

Lemma 2. Suppose that, for some $m \in \mathbb{Z}$,

$$
\sum_{k=-\infty}^{m} \max \left(q^{k}, q^{\alpha k}\right)\left|u\left(q^{k}\right)\right|<\infty \quad \text { if } \alpha \neq 1,
$$

and

$$
\sum_{k=-\infty}^{m}|k| q^{k}\left|u\left(q^{k}\right)\right|<\infty \quad \text { if } \alpha=1 .
$$

Then $I^{\alpha} u$ exists, it is a radial function, and for any $x \neq 0$, we have

$$
\begin{aligned}
& \left(I^{\alpha} u\right)\left(|x|_{K}\right)= \\
& \quad q^{-\alpha}|x|_{K}^{\alpha} u\left(|x|_{K}\right)+\frac{1-q^{-\alpha}}{1-q^{\alpha-1}} \int_{|y|_{K}<|x|_{K}}\left(|x|_{K}^{\alpha-1}-|y|_{K}^{\alpha-1}\right) u\left(|y|_{K}\right) d y
\end{aligned}
$$

if $\alpha \neq 1$, and 


$$
\begin{aligned}
& \left(I^{1} u\right)\left(|x|_{K}\right)= \\
& \quad q^{-1}|x|_{K} u\left(|x|_{K}\right)+\frac{1-q}{q \log q} \int_{|y|_{K}<|x|_{K}}\left(\log |x|_{K}-\log |y|_{K}\right) u\left(|y|_{K}\right) d y .
\end{aligned}
$$

Proof. It is sufficient to compute the integrals over the set $\left\{y \in K:|y|_{K}=|x|_{K}\right\}$, and that is done using the integration formulas (2-3) and (2-5).

It follows from Lemma 2 that the function $I^{\alpha} u$ is continuous if, for example, $u$ is bounded near the origin (see an estimate of the integral $I_{\alpha, 0}$ in the proof of Theorem 1 below). If $\left|u\left(|x|_{K}\right)\right| \leq C|x|_{K}^{-\varepsilon}$, as $|x|_{K} \geq 1$, then $\left|\left(I^{\alpha} u\right)\left(|x|_{K}\right)\right| \leq C|x|_{K}^{\alpha-\varepsilon}$, as $|x|_{K} \geq 1$. Here and below we denote by $C$ various (possibly different) positive constants.

It is easy to transform (3-10) and (3-11) further obtaining series involving $u\left(q^{n}\right)$.

Obviously, $D^{\alpha} I^{\alpha}=I$ on $\mathscr{D}(K)$, if $\alpha \neq 1$, and on $\Phi(K)$, if $\alpha=1$. Since by Lemma 1 and Lemma 2, the operators are defined in a straightforward sense for wider classes of functions, it is natural to look for conditions sufficient for this identity.

Lemma 3. Suppose that for some $m \in \mathbb{Z}$,

$$
\begin{aligned}
& \sum_{k=-\infty}^{m} \max \left(q^{k}, q^{\alpha k}\right)\left|v\left(q^{k}\right)\right|<\infty \text { and } \sum_{l=m}^{\infty}\left|v\left(q^{l}\right)\right|<\infty \quad \text { if } \alpha \neq 1 \text {, } \\
& \sum_{k=-\infty}^{m}|k| q^{k}\left|v\left(q^{k}\right)\right|<\infty \quad \text { and } \quad \sum_{l=m}^{\infty} l\left|v\left(q^{l}\right)\right|<\infty \quad \text { if } \alpha=1 .
\end{aligned}
$$

Then there exists $\left(D^{\alpha} I^{\alpha} v\right)\left(|x|_{K}\right)=v\left(|x|_{K}\right)$ for any $x \neq 0$.

The proof consists of tedious but quite elementary calculations based on the integration formulas (2-2)-(2-8). A relatively nontrivial tool is the sum formula for the arithmetic-geometric progression (from [Gradshteyn and Ryzhik 1996, Formula 0.113]).

Using Lemma 3, we can consider the simplest Cauchy problem

$$
D^{\alpha} u\left(|x|_{K}\right)=f\left(|x|_{K}\right), \quad u(0)=0,
$$

where $f$ is a continuous function, such that

$$
\sum_{l=m}^{\infty}\left|f\left(q^{l}\right)\right|<\infty, \text { if } \alpha \neq 1, \quad \text { or } \quad \sum_{l=m}^{\infty} l\left|f\left(q^{l}\right)\right|<\infty, \text { if } \alpha=1 .
$$

The unique strong solution is $u=I^{\alpha} f$; the uniqueness follows from the fact that the equality $D^{\alpha} u=0$ (in the sense of $\mathscr{D}^{\prime}(K)$ ) implies the equality $u=$ const; see [Vladimirov et al. 1994] or [Kochubei 2001]. Therefore on radial functions, the operators $D^{\alpha}$ and $I^{\alpha}$ behave like the Caputo-Dzhrbashyan fractional derivative 
and the Riemann-Liouville fractional integral of real analysis (see, for example, [Kilbas et al. 2006]). However the next example illustrates a different behavior of the "fractional integral" in the non-Archimedean case.

Example. Let $f\left(|x|_{K}\right) \equiv 1, x \in K$. Then $\left(I^{\alpha} f\right)\left(|x|_{K}\right) \equiv 0$.

Proof. Let $|x|_{K}=q^{n}$. If $\alpha \neq 1$, then by (3-10), (2-2), and (2-6),

$$
\begin{aligned}
\left(I^{\alpha} f\right)\left(|x|_{K}\right) & =q^{-\alpha}|x|_{K}^{\alpha}+\frac{1-q^{-\alpha}}{1-q^{\alpha-1}} \int_{|y|_{K} \leq q^{n-1}}\left(|x|_{K}^{\alpha-1}-|y|_{K}^{\alpha-1}\right) d y \\
& =q^{-\alpha}|x|_{K}^{\alpha}+\frac{1-q^{-\alpha}}{1-q^{\alpha-1}}\left[q^{n-1}|x|_{K}^{\alpha-1}-\frac{1-q^{-1}}{1-q^{-\alpha}} q^{\alpha(n-1)}\right] \\
& =q^{-\alpha}|x|_{K}^{\alpha}+\frac{1-q^{-\alpha}}{1-q^{\alpha-1}}|x|_{K}^{\alpha} \frac{q^{-1}-q^{-\alpha}}{1-q^{-\alpha}}=0 .
\end{aligned}
$$

If $\alpha=1$, then by (3-11), (2-4), and (2-6),

$$
\begin{aligned}
\left(I^{1} f\right)\left(|x|_{K}\right) & =q^{-1}|x|_{K}+\frac{1-q}{q \log q} \int_{|y|_{K} \leq q^{n-1}}\left(\log |x|_{K}-\log |y|_{K}\right) d y \\
& =q^{-1}|x|_{K}+\frac{1-q}{q \log q}\left[q^{n-1} \log |x|_{K}-\left(n-1-\frac{1}{q-1}\right) q^{n-1} \log q\right] \\
& =|x|_{K}\left(q^{-1}+\frac{1-q}{q \log q}\left(1+\frac{1}{q-1}\right) q^{-1} \log q\right)=0 .
\end{aligned}
$$

Of course, these identities in the weaker sense of distributions from $\Phi^{\prime}(K)$ are trivial, since the constant functions are identified with zero, $I^{\alpha}$ with $D^{-\alpha}$, and $D^{\alpha} D^{-\alpha}=I$.

On the other hand, the example shows that the condition of decay at infinity in Lemma 3 cannot be dropped.

\section{Fractional differential equations}

4.1. The Cauchy problem and an integral equation. In the class of radial functions $u=u\left(|x|_{K}\right)$, we consider the Cauchy problem

$$
\begin{aligned}
D^{\alpha} u+a\left(|x|_{K}\right) u & =f\left(|x|_{K}\right), \quad x \in K, \\
u(0) & =0,
\end{aligned}
$$

where $a$ and $f$ are continuous functions, that is, they have finite limits $a(0)$ and $f(0)$, as $x \rightarrow 0$.

Looking for a solution of the form $u=I^{\alpha} v$, where $v$ is a radial function, we obtain formally an integral equation

$$
v\left(|x|_{K}\right)+a\left(|x|_{K}\right)\left(I^{\alpha} v\right)\left(|x|_{K}\right)=f\left(|x|_{K}\right), \quad x \in K .
$$


Let us study its solvability. Later we investigate, in what sense a solution of (4-3) corresponds to a solution of the Cauchy problem (4-1)-(4-2).

It follows from (4-3) that $v(0)=f(0)$. Suppose first that $\alpha \neq 1$. By Lemma 2, (4-3) can be written in the form

$$
\begin{aligned}
& {\left[1+q^{-\alpha} a\left(|x|_{K}\right)|x|_{K}^{\alpha}\right] v\left(|x|_{K}\right)} \\
& \quad+c_{\alpha} a\left(|x|_{K}\right) \int_{|y|_{K}<|x|_{K}}\left(|x|_{K}^{\alpha-1}-|y|_{K}^{\alpha-1}\right) v\left(|y|_{K}\right) d y=f\left(|x|_{K}\right), \quad x \neq 0,
\end{aligned}
$$

where $c_{\alpha}=\left(1-q^{-\alpha}\right) /\left(1-q^{\alpha-1}\right)$.

Since $a$ is continuous, there exists such $N \in \mathbb{Z}$ that

$$
q^{-\alpha} a\left(|x|_{K}\right)|x|_{K}^{\alpha}<1 \quad \text { for }|x|_{K} \leq q^{N} .
$$

On the ball $B_{N}=\left\{x \in K:|x|_{K} \leq q^{N}\right\}$, the equation takes the form

$$
v\left(|x|_{K}\right)+\int_{|y|_{K}<|x|_{K}} k_{\alpha}(x, y) v\left(|y|_{K}\right) d y=F\left(|x|_{K}\right)
$$

where

$$
k_{\alpha}(x, y)=\left\{\begin{array}{cl}
{\left[1+q^{-\alpha} a\left(|x|_{K}\right)|x|_{K}^{\alpha}\right]^{-1} c_{\alpha} a\left(|x|_{K}\right)\left(|x|_{K}^{\alpha-1}-|y|_{K}^{\alpha-1}\right)} & \text { if } x \neq 0, \\
0 & \text { if } x=0,
\end{array}\right.
$$

and

$$
F\left(|x|_{K}\right)=\left[1+q^{-\alpha} a\left(|x|_{K}\right)|x|_{K}^{\alpha}\right]^{-1} f\left(|x|_{K}\right) .
$$

If we construct a solution of (4-4) on $B_{N}$, and if

$$
a\left(|x|_{K}\right) \neq-q^{\alpha m} \quad \text { for any } x \in K, m \in \mathbb{Z},
$$

we will be able to construct a solution of (4-4), thus a solution of (4-3), successively for all $x \in K$.

If $\alpha=1$, we use (3-11) and obtain in a similar way the equation (4-4) with $k_{1}(x, y)=\left\{\begin{array}{cl}\frac{1-q}{q \log q}\left[1+q^{-1} a\left(|x|_{K}\right)|x|_{K}\right]^{-1} a\left(|x|_{K}\right)\left(\log |x|_{K}-\log |y|_{K}\right) & \text { if } x \neq 0, \\ 0 & \text { if } x=0,\end{array}\right.$ and $F\left(|x|_{K}\right)=\left[1+q^{-1} a\left(|x|_{K}\right)|x|_{K}\right]^{-1} f\left(|x|_{K}\right)$.

It is convenient to extend $k_{\alpha}$ (including the case $\alpha=1$ ) by zero onto $B_{N} \times B_{N}$. Theorem 1. For each $\alpha>0$, the integral equation (4-4) has a unique continuous solution on $B_{N}$. 
Proof. Let us consider the integral operator $\mathscr{K}$ appearing in (4-4) as an operator on the Banach space $C\left(B_{N}\right)$ of complex-valued continuous functions on $B_{N}$. By the theory of integral operators developed in sufficient generality in [Edwards 1965] (see Proposition 9.5.17), to prove that $\mathscr{K}$ is a compact operator, it suffices to check that, for any $x_{0} \in B_{N}$,

$$
\lim _{x \rightarrow x_{0}} \int_{B_{N}}\left|k_{\alpha}(x, y)-k_{\alpha}\left(x_{0}, y\right)\right| d y=0 .
$$

The relation (4-6) is obvious for $x_{0} \neq 0$, and also for $\alpha>1$. For $x_{0}=0$, we have $k_{\alpha}(0, y)=0$, and for $0<\alpha<1,|x|_{K}=q^{n}, n \geq N$, we get by (2-2) and (2-6) that

$$
\begin{aligned}
\int_{B_{N}}\left|k_{\alpha}(x, y)\right| d y & =\text { const } \int_{|y|_{K} \leq q^{n-1}}\left(|y|_{K}^{\alpha-1}-q^{n(\alpha-1)}\right) d y \\
& =\operatorname{const}\left(\frac{1-q^{-1}}{1-q^{-\alpha}} q^{\alpha(n-1)}-q^{n(\alpha-1)} q^{n-1}\right)=\mathrm{const}|x|_{K}^{\alpha},
\end{aligned}
$$

which tends to 0 as $|x|_{K} \rightarrow 0$. For $\alpha=1$, we use (2-4) and (2-6) to obtain that

$$
\begin{aligned}
\int_{B_{N}}\left|k_{1}(x, y)\right| d y & =\text { const } \int_{|y|_{K} \leq q^{n-1}}\left(\log q^{n}-\log |y|_{K}\right) d y \\
& =\text { const }\left(n q^{n-1} \log q-\left(n-1-\frac{1}{q-1}\right) q^{n-1} \log q\right) \\
& =\text { const } \frac{q}{q-1} q^{n-1} \log q=\mathrm{const} \frac{\log q}{q-1}|x|_{K},
\end{aligned}
$$

and this again tends to 0 as $|x|_{K} \rightarrow 0$.

Thus, $\mathscr{K}$ is compact, and by the Fredholm alternative [Edwards 1965, 9.10.3], our theorem will be proved if we show that $\mathscr{K}$ has no nonzero eigenvalues.

Suppose that $\mathscr{K} w=\lambda w, \lambda \neq 0$, for some $w \in C\left(B_{N}\right)$. We have $|w(y)| \leq C$,

$$
\left|k_{\alpha}(x, y)\right| \leq\left. M|| x\right|_{K} ^{\alpha-1}-|y|_{K}^{\alpha-1} \mid,
$$

if $\alpha \neq 1$, and

$$
\left|k_{1}(x, y)\right| \leq M\left(\log |x|_{K}-\log |y|_{K}\right),
$$

if $\alpha=1,|y|_{K}<|x|_{K}$.

In subsequent iterations we will deal with the integrals

$$
\begin{aligned}
I_{\alpha, m} & =\left.\int_{|y|_{K}<|x|_{K}}|| x\right|_{K} ^{\alpha-1}-\left.|y|_{K}^{\alpha-1}|| y\right|_{K} ^{\alpha m} d y, \quad \alpha \neq 1, \\
I_{1, m} & =\int_{|y|_{K}<|x|_{K}}\left(\log |x|_{K}-\log |y|_{K}\right)|y|_{K}^{m} d y .
\end{aligned}
$$


If $\alpha>1$, we find denoting $|x|_{K}=q^{n}$ and using (2-2) that

$$
I_{\alpha, m}=|x|_{K}^{\alpha-1} \int_{|y|_{K} \leq q^{n-1}}|y|_{K}^{\alpha m} d y-\int_{|y|_{K} \leq q^{n-1}}|y|_{K}^{\alpha(m+1)-1} d y=d_{\alpha, m}|x|_{K}^{\alpha(m+1)}
$$

where, for all $m=0,1,2, \ldots$

$$
\begin{aligned}
d_{\alpha, m} & =\frac{1-q^{-1}}{1-q^{-\alpha m-1}} q^{-\alpha m-1}-\frac{1-q^{-1}}{1-q^{-\alpha m-\alpha}} q^{-\alpha m-\alpha} \\
& =\left(1-q^{-1}\right) \frac{q^{\alpha-1}-1}{\left(1-q^{-\alpha m-1}\right)\left(q^{\alpha m+\alpha}-1\right)} \leq A q^{-\alpha m}
\end{aligned}
$$

for some $A>0$. A similar result,

$$
I_{\alpha, m}=d_{\alpha, m}|x|_{K}^{\alpha(m+1)}, \quad d_{\alpha, m} \leq A q^{-\alpha m}, \quad m=0,1,2, \ldots
$$

is obtained for $0<\alpha<1$, so that (4-7) holds for any $\alpha \neq 1$. If $\alpha=1$, then the integral $I_{1, m}$ is evaluated as follows. We have

$$
\begin{aligned}
I_{1, m} & =\sum_{k=-\infty}^{n-1} \int_{|y|_{K}=q^{k}}\left(\log |x|_{K}-\log |y|_{K}\right)|y|_{K}^{m} d y \\
& =\left(1-\frac{1}{q}\right) \log q \sum_{k=-\infty}^{n-1}(n-k) q^{k(m+1)} \\
& =\left(1-\frac{1}{q}\right) \log q \sum_{\nu=1}^{\infty} v q^{(n-v)(m+1)}=d_{1, m}|x|_{K}^{\alpha(m+1)}
\end{aligned}
$$

where

$$
d_{1, m}=\left(1-\frac{1}{q}\right) \log q \sum_{\nu=1}^{\infty} v q^{-v(m+1)}=\left(1-\frac{1}{q}\right) \log q \frac{q^{-m-1}}{\left(1-q^{-m-1}\right)^{2}} \leq A q^{-m}
$$

(we have used [Gradshteyn and Ryzhik 1996, Identity 0.231.2]). Thus, we have proved (4-7) also for $\alpha=1$.

Let us return to a function $w$ satisfying the relation $\mathscr{T} w=\lambda w, \lambda \neq 0$. Using (4-7) (separately for $\alpha \neq 1$ and $\alpha=1$ ) and iterating we find by induction that

$$
|w(x)| \leq C\left(M|\lambda|^{-1} A\right)^{m}\left(\prod_{j=0}^{m} q^{-\alpha j}\right)|x|_{K}^{\alpha m}, \quad m=0,1,2, \ldots, x \in B_{N} .
$$

Since $\prod_{j=0}^{m} q^{-\alpha j}=q^{-\frac{\alpha}{2} m(m+1)}$, it follows from (4-8) that $w(x) \equiv 0$.

4.2. Strong solutions. Below we assume that the inequality (4-5) is satisfied. Then, as we have mentioned, the solution $v$ of (4-4) is automatically extended in a unique way from $B_{N}$ onto $K$. The extended function $v$ satisfies (4-3). Therefore the 
function $u=I^{\alpha} v$ satisfies (4-1) in the sense of distributions from $\Phi^{\prime}$. The initial condition (4-2) is satisfied automatically.

Let us find additional conditions on $a$ and $f$, under which this construction gives a strong solution of the Cauchy problem (4-1)-(4-2). Note that, by Lemma 3 and Theorem 1 , a strong solution is unique in the class of functions $u=I^{\alpha} v$ where $v$ is a continuous radial function, such that $\sum_{l=m}^{\infty}\left|v\left(q^{l}\right)\right|<\infty$ for some $m \in \mathbb{Z}$.

\section{Theorem 2. Suppose that}

$$
\left|a\left(|x|_{K}\right)\right| \leq C|x|_{K}^{-\alpha-\varepsilon}, \quad\left|f\left(|x|_{K}\right)\right| \leq C|x|_{K}^{-\varepsilon}, \quad \varepsilon>0, C>0,
$$

as $|x|_{K}>1$. Then $u=I^{\alpha} v$ is a strong solution of the Cauchy problem (4-1)-(4-2).

Proof. Let $v\left(|x|_{K}\right)$ be the solution of (4-3') constructed above for all $x \in K$ (for $x=0$, the integral in the right-hand side is assumed equal to zero). For $|x|_{K} \leq q^{N}$ the existence of a solution $v$ was obtained from the theory of compact operators; for larger values of $|x|_{K}$ we use successively (4-3') itself. Denote

$$
V_{m}=\sup _{|x|_{K} \leq q^{m}}\left|v\left(q^{m}\right)\right|
$$

The sequence $\left\{V_{m}\right\}$ is nondecreasing.

As we assumed in Theorem 1 only the continuity of the coefficient $a$, we took $N$ in such a way that the neighborhood $B_{N}=\left\{x:|x|_{K} \leq q^{N}\right\}$ was sufficiently small. Here we assume (4-5), so that we can take any fixed integer $N$ and obtain a solution $v$ on $B_{N}$.

Consider the case where $\alpha \neq 1$. It follows from (4-5) and (4-9) that

$$
\left|\left[1+q^{-\alpha} a\left(|x|_{K}\right)|x|_{K}^{\alpha}\right]^{-1}\right| \leq H
$$

where $H>0$ does not depend on $x \in K$. If $m \geq N$, then we find from (4-3') and the above estimate for $I_{\alpha, 0}$ that

$$
\left|v\left(q^{m}\right)\right| \leq c_{\alpha} d_{\alpha, 0} H a\left(q^{m}\right) q^{\alpha m} V_{m-1}+H\left|f\left(g^{m}\right)\right| .
$$

Let us choose $m_{1} \geq N$ so big that

$$
c_{\alpha} d_{\alpha, 0} H a\left(q^{m}\right) q^{\alpha m} \leq \frac{1}{2}, \quad H\left|f\left(g^{m}\right)\right| \leq \frac{1}{2} V_{N-1},
$$

as $m \geq m_{1}$ (that is possible due to (4-9)). Then it follows from (4-10) that $V_{m} \leq V_{m-1}$, as $m \geq m_{1}$, hence that the function $v$ is bounded on $K$.

Now we get from (4-3') and the assumptions (4-9) that

$$
\left|v\left(|x|_{K}\right)\right| \leq C|x|_{K}^{-\varepsilon}, \quad|x|_{K} \geq 1,
$$

$C>0$. A similar reasoning works for $\alpha=1$. 
Taking into account the estimate (4-11) we find from Lemma 3 that

$$
\left(D^{\alpha} I^{\alpha} v\right)\left(|x|_{K}\right)=v\left(|x|_{K}\right), \quad x \neq 0 .
$$

Therefore the function $u=I^{\alpha} v$ satisfies (4-1) for all $x \neq 0$. Since $a, f$, and $u$ are continuous, the equation is satisfied in the strong sense.

4.3. Generalizations. Instead of (4-2), one can consider an inhomogeneous initial condition $u(0)=u_{0}, u_{0} \in \mathbb{C}$. Looking for a solution in the form $u=u_{0}+I^{\alpha} v$, $v=v\left(|x|_{K}\right)$, we obtain the integral equation

$$
v\left(|x|_{K}\right)+a\left(|x|_{K}\right)\left(I^{\alpha} v\right)\left(|x|_{K}\right)=f\left(|x|_{K}\right)-a\left(|x|_{K}\right) u_{0},
$$

which can be studied under the same assumptions.

All the above results carry over to the case of a matrix-valued coefficient $a\left(|x|_{K}\right)$ and vector-valued solutions. In this case, to obtain a strong solution, it is sufficient to demand that the spectrum of each matrix $a\left(|x|_{K}\right), x \in K$, does not intersect the set $\left\{-q^{N}: N \in \mathbb{Z}\right\}$.

\section{Acknowledgement}

The author is grateful to M. M. Malamud for a helpful discussion.

\section{References}

[Albeverio et al. 2010] S. Albeverio, A. Y. Khrennikov, and V. M. Shelkovich, Theory of p-adic distributions: Linear and nonlinear models, London Mathematical Society Lecture Note Series 370, Cambridge University Press, 2010. MR 2011f:46038 Zbl 1198.46001

[Edwards 1965] R. E. Edwards, Functional analysis: Theory and applications, Holt, Rinehart and Winston, New York, 1965. MR 36 \#4308 Zbl 0182.16101

[Gradshteyn and Ryzhik 1996] I. S. Gradshteyn and I. M. Ryzhik, Table of integrals, series, and products, 5th ed., Academic Press, San Diego, 1996. MR 97c:00014 Zbl 0918.65001

[Kilbas et al. 2006] A. A. Kilbas, H. M. Srivastava, and J. J. Trujillo, Theory and applications of fractional differential equations, North-Holland Mathematics Studies 204, Elsevier, Amsterdam, 2006. MR 2007a:34002 Zbl 1092.45003

[Kochubei 2001] A. N. Kochubei, Pseudo-differential equations and stochastics over non-Archimedean fields, Monographs and Textbooks in Pure and Applied Mathematics 244, Marcel Dekker, New York, 2001. MR 2003b:35220 Zbl 0984.11063

[Kochubei 2008] A. N. Kochubei, "A non-Archimedean wave equation”, Pacific J. Math. 235:2 (2008), 245-261. MR 2009e:35305 Zbl 1190.35235

[Vladimirov 2003] V. S. Vladimirov, Tables of integrals of complex-valued functions of p-adic arguments, Current Problems in Mathematics 2, Steklov Mathematical Institute, Moscow, 2003. In Russian; translated in ArXiv: math-ph/9911027. MR 2006a:11164 Zbl 1062.11003

[Vladimirov et al. 1994] V. S. Vladimirov, I. V. Volovich, and E. I. Zelenov, p-adic analysis and mathematical physics, Series on Soviet and East European Mathematics 1, World Scientific, River Edge, NJ, 1994. MR 95k:11155 Zbl 0812.46076 
[Zúñiga-Galindo 2011] W. A. Zúñiga-Galindo, "Local zeta functions and fundamental solutions for pseudo-differential operators over $p$-adic fields", p-Adic Numbers Ultrametric Anal. Appl. 3:4 (2011), 344-358. MR 2854684 Zbl 06105091

Received February 20, 2013.

ANATOLY N. KochubeI

INSTITUTE OF MATHEMATICS

National ACADEMy of ScIENCES OF UKRAine

TERESHCHENKIVSKA 3

KIEV, 01601

UKRAINE

kochubei@i.com.ua 


\title{
PACIFIC JOURNAL OF MATHEMATICS
}

\author{
msp.org/pjm
}

Founded in 1951 by E. F. Beckenbach (1906-1982) and F. Wolf (1904-1989)

\section{EDITORS}

Don Blasius (Managing Editor)

Department of Mathematics

University of California

Los Angeles, CA 90095-1555

blasius@math.ucla.edu

\author{
Paul Balmer \\ Department of Mathematics \\ University of California \\ Los Angeles, CA 90095-1555 \\ balmer@math.ucla.edu \\ Robert Finn \\ Department of Mathematics \\ Stanford University \\ Stanford, CA 94305-2125 \\ finn@math.stanford.edu \\ Sorin Popa \\ Department of Mathematics \\ University of California \\ Los Angeles, CA 90095-1555 \\ popa@math.ucla.edu
}

\author{
Vyjayanthi Chari \\ Department of Mathematics \\ University of California \\ Riverside, CA 92521-0135 \\ chari@math.ucr.edu \\ Kefeng Liu \\ Department of Mathematics \\ University of California \\ Los Angeles, CA 90095-1555 \\ liu@math.ucla.edu \\ Jie Qing \\ Department of Mathematics \\ University of California \\ Santa Cruz, CA 95064 \\ qing@ cats.ucsc.edu
}

\section{PRODUCTION}

Silvio Levy, Scientific Editor, production@msp.org

\section{SUPPORTING INSTITUTIONS}

ACADEMIA SINICA, TAIPEI

CALIFORNIA INST. OF TECHNOLOGY

INST. DE MATEMÁTICA PURA E APLICADA

KEIO UNIVERSITY

MATH. SCIENCES RESEARCH INSTITUTE

NEW MEXICO STATE UNIV.

OREGON STATE UNIV.

\author{
STANFORD UNIVERSITY \\ UNIV. OF BRITISH COLUMBIA \\ UNIV. OF CALIFORNIA, BERKELEY \\ UNIV. OF CALIFORNIA, DAVIS \\ UNIV. OF CALIFORNIA, LOS ANGELES \\ UNIV. OF CALIFORNIA, RIVERSIDE \\ UNIV. OF CALIFORNIA, SAN DIEGO \\ UNIV. OF CALIF., SANTA BARBARA
}

\author{
Daryl Cooper \\ Department of Mathematics \\ University of California \\ Santa Barbara, CA 93106-3080 \\ cooper@math.ucsb.edu \\ Jiang-Hua Lu \\ Department of Mathematics \\ The University of Hong Kong \\ Pokfulam Rd., Hong Kong \\ jhlu@maths.hku.hk \\ Paul Yang \\ Department of Mathematics \\ Princeton University \\ Princeton NJ 08544-1000 \\ yang@math.princeton.edu
}

These supporting institutions contribute to the cost of publication of this Journal, but they are not owners or publishers and have no responsibility for its contents or policies.

See inside back cover or msp.org/pjm for submission instructions.

The subscription price for 2014 is US $\$ 410 /$ year for the electronic version, and \$535/year for print and electronic.

Subscriptions, requests for back issues and changes of subscribers address should be sent to Pacific Journal of Mathematics, P.O. Box 4163, Berkeley, CA 94704-0163, U.S.A. The Pacific Journal of Mathematics is indexed by Mathematical Reviews, Zentralblatt MATH, PASCAL CNRS Index, Referativnyi Zhurnal, Current Mathematical Publications and Web of Knowledge (Science Citation Index).

The Pacific Journal of Mathematics (ISSN 0030-8730) at the University of California, c/o Department of Mathematics, 798 Evans Hall \#3840, Berkeley, CA 94720-3840, is published twelve times a year. Periodical rate postage paid at Berkeley, CA 94704, and additional mailing offices. POSTMASTER: send address changes to Pacific Journal of Mathematics, P.O. Box 4163, Berkeley, CA 94704-0163.

PJM peer review and production are managed by EditFLOW ${ }^{\circledR}$ from Mathematical Sciences Publishers.

\section{PUBLISHED BY}

\section{mathematical sciences publishers \\ nonprofit scientific publishing}

http://msp.org/

(C) 2014 Mathematical Sciences Publishers 


\section{PACIFIC JOURNAL OF MATHEMATICS}

Volume $269 \quad$ No. $2 \quad$ June 2014

Totaro's question for simply connected groups of low rank

257

JODI BLACK and RAMAN PARIMALA

Uniform hyperbolicity of the curve graphs

269

BRIAN H. BOWDITCH

Constant Gaussian curvature surfaces in the 3-sphere via loop groups

281

DAVID BRANDER, JUN-ICHI INOGUCHI and SHIMPEI KOBAYASHI

On embeddings into compactly generated groups

305

PIERRE-EMmanuel CAPRACE and Yves CORNULIER

Variational representations for $N$-cyclically monotone vector fields

ALFRED GALICHON and NASSIF GHOUSSOUB

Restricted successive minima

341

MaRTIN HenK and CARSTEN THIEL

Radial solutions of non-Archimedean pseudodifferential equations

355

ANATOLY N. KochubeI

A Jantzen sum formula for restricted Verma modules over affine Kac-Moody

algebras at the critical level

JOHANNES KÜBEL

Notes on the extension of the mean curvature flow

YAN LENG, ENTAO ZHAO and HaORAN ZHAO

Hypersurfaces with prescribed angle function

Henrique F. DE Lima, ERAldo A. Lima JR. and Ulisses L. Parente

Existence of nonparametric solutions for a capillary problem in warped products

JoRGE H. LiRA and GABRIELA A. WANDERLEY

A counterexample to the simple loop conjecture for $\operatorname{PSL}(2, \mathbb{R})$

425

KATHRYN MANN

Twisted Alexander polynomials of 2-bridge knots for parabolic representations

TAKAYUKI MORIFUJI and ANH T. TRAN

Schwarzian differential equations associated to Shimura curves of genus zero

FANG-TING TU

Polynomial invariants of Weyl groups for Kac-Moody groups 